\title{
Increased MAPK reactivation in early resistance to dabrafenib/trametinib combination therapy of BRAF-mutant metastatic melanoma
}

Georgina V. Long1,2,3, Carina Fung ${ }^{4,5}$, Alexander M. Menzies ${ }^{1,2,6}$, Gulietta M. Pupo5 , Matteo S. Carlino ${ }^{1,5,6}$, Jessica Hyman ${ }^{1,7}$, Hamideh Shahheydari ${ }^{4,5}$, Varsha Tembe ${ }^{5}$, John F. Thompson ${ }^{1,8,9}$, Robyn P. Saw ${ }^{1,8,9}$, Julie Howle ${ }^{1,8,10}$, Nicholas K. Hayward ${ }^{11}$, Peter Johansson ${ }^{11}$, Richard A. Scolyer ${ }^{1,7,12}$, Richard F. Kefford ${ }^{1,2,4,5}$ $\&$ Helen Rizos ${ }^{4,5}$

One-third of BRAF-mutant metastatic melanoma patients treated with combined BRAF and MEK inhibition progress within 6 months. Treatment options for these patients remain limited. Here we analyse $20 B R A F^{\mathrm{V} 600}$-mutant melanoma metastases derived from 10 patients treated with the combination of dabrafenib and trametinib for resistance mechanisms and genetic correlates of response. Resistance mechanisms are identified in 9/11 progressing tumours and MAPK reactivation occurred in 9/10 tumours, commonly via BRAF amplification and mutations activating NRAS and MEK2. Our data confirming that MEK2 ${ }^{\mathrm{C} 125 \mathrm{~S}}$, but not the synonymous MEK1 ${ }^{\mathrm{C} 1215}$ protein, confers resistance to combination therapy highlight the functional differences between these kinases and the preponderance of MEK2 mutations in combination therapy-resistant melanomas. Exome sequencing did not identify additional progression-specific resistance candidates. Nevertheless, most melanomas carried additional oncogenic mutations at baseline (for example, RAC1 and AKT3) that activate the MAPK and PI3K pathways and are thus predicted to diminish response to MAPK inhibitors.

\footnotetext{
${ }^{1}$ Melanoma Institute Australia, Sydney, New South Wales 2060, Australia. ${ }^{2}$ Discipline of Medicine, Sydney Medical School, The University of Sydney, Sydney, New South Wales 2006, Australia. ${ }^{3}$ Mater Hospital, North Sydney, New South Wales 2060, Australia. ${ }^{4}$ Precision Cancer Therapy Laboratory, Australian School of Advanced Medicine, Macquarie University, Sydney, New South Wales 2109, Australia. ${ }^{5}$ Westmead Institute for Cancer Research, The University of Sydney at Westmead Millennium Institute, Westmead Hospital, Westmead, New South Wales 2145, Australia. ${ }^{6}$ Department of Medical Oncology, Crown Princess Mary Cancer Centre, Westmead Hospital, Westmead, New South Wales 2145, Australia. ${ }^{7}$ Departments of Tissue Pathology and Diagnostic Oncology, Royal Prince Alfred Hospital, Camperdown, New South Wales 2006, Australia. ${ }^{8}$ Discipline of Surgery, Sydney Medical School, The University of Sydney, Sydney, New South Wales 2006, Australia. ${ }^{9}$ Departments of Melanoma and Surgical Oncology, Royal Prince Alfred Hospital, Camperdown, New South Wales 2006, Australia. ${ }^{10}$ Department of Surgical Oncology, Crown Princess Mary Cancer Centre, Westmead Hospital, Westmead, New South Wales 2145, Australia. ${ }^{11}$ Oncogenomics Laboratory, QIMR Berghofer Medical Research Institute, Herston, Brisbane, Queensland 4006, Australia. ${ }^{12}$ Discipline of Pathology, Sydney Medical School, The University of Sydney, Sydney, New South Wales 2006, Australia. Correspondence and requests for materials should be addressed to H.R. (email: helen.rizos@mq.edu.au).
} 
T herapy targeting the mitogen-activated protein kinase (MAPK) pathway with combined BRAF and MEK inhibition was recently approved by the Australian Therapeutic Goods Administration, and had accelerated approval by the USA Food and Drug Administration for the treatment of $\mathrm{BRAF}^{\mathrm{V} 600}$-mutant metastatic melanoma. The randomized phase II study of the BRAF inhibitor dabrafenib combined with the MEK inhibitor trametinib significantly improved response rates (76 versus 54\%), prolonged progression-free survival (PFS; 9.4 versus 5.8 months) and reduced skin toxicities compared with single-agent dabrafenib, in patients with $\mathrm{BRAF}^{\mathrm{V} 600}$-mutant metastatic melanoma ${ }^{1}$. The phase III study of this combination versus dabrafenib (NCT01584648) demonstrated an improved PFS in the combination-treated cohort (hazard ratio $0.75, P=0.035)^{2}$ and a phase III trial demonstrated an overall survival (OS) benefit for this combination compared with vemurafenib monotherapy (NCT01597908) ${ }^{3}$. Other BRAF and MEK inhibitor combinations, including vemurafenib combined with cobimetinib ${ }^{4}$ (NCT01689519) and encorafenib (LGX818) combined with binimetinib (MEK162; ref. 5; NCT01909453), have been or are also being evaluated in ongoing clinical trials in $\mathrm{BRAF}^{\mathrm{V} 600}$-mutant melanoma patients.

Despite significant improvements in clinical outcomes and reports of long-term responses ${ }^{6}, 50 \%$ of patients treated with dabrafenib combined with trametinib progress at 9-10 months, and resistance remains a barrier to better patient outcomes. Acquired resistance to BRAF inhibitor monotherapy is primarily driven by MAPK reactivation ${ }^{7-9}$, via $B R A F$ copy number gains, aberrant BRAF splicing, mutations in NRAS or MEK1/2 and upregulation of receptor tyrosine kinases ${ }^{10-13}$.

The role of MAPK signalling and the spectrum of alterations conferring resistance to combined BRAF and MEK inhibition remain unknown. Given that many BRAF inhibitor resistance mechanisms are sensitive to MEK inhibition, alone or in combination with BRAF inhibition ${ }^{7,10,14,15}$, MAPK-independent mechanisms of resistance might be anticipated to predominate in melanomas treated with combined BRAF/MEK inhibition.
However, a study of five BRAF inhibitor-naive patients with acquired resistance to combined BRAF and MEK inhibition identified potential resistance mechanisms in three patients, including a MEK2 ${ }^{\mathrm{Q} 60 \mathrm{P}}$ mutation, amplification of the $B R A F$ gene and expression of a BRAF-splice variant lacking exons 2-10 $\left(B R A F^{\operatorname{ex} 2-10 \Delta}\right)^{16}$. The MEK2 ${ }^{\mathrm{Q} 60 \mathrm{P}}$ mutation and BRAF amplification have been shown to confer resistance to combined BRAF and MEK inhibition in preclinical models ${ }^{16}$. BRAF-splice variants, however, remain sensitive to MEK inhibition $^{7,12,14}$, thus the contribution of BRAF $^{\operatorname{ex} 2-10 \Delta}$ in combination BRAF/MEK resistance remains unclear.

Defining the mechanisms of acquired resistance to combined BRAF and MEK inhibition and how they may differ from those observed with single-agent BRAF inhibition may provide an opportunity for therapeutic exploitation, particularly in patients who show minimal clinical benefit. Here we describe resistance mechanisms in 20 melanoma samples ( 9 pre-treatment and 11 progressing (Prog) metastases) derived from $10 \mathrm{BRAF}^{\mathrm{V} 600}$ mutant melanoma patients who had early resistance to combined BRAF and MEK inhibitor therapy.

\section{Results}

Patients and melanoma tissue samples. Eleven Prog and nine matched pre-treatment melanoma tumour samples derived from $10 \mathrm{BRAF}^{\nabla 600}$-mutant melanoma patients treated with dabrafenib plus trametinib (CombiDT) were analysed (Table 1). Two patients received prior MAPK pathway inhibitors; patient $\mathrm{C} 10$ received dabrafenib monotherapy and patient $\mathrm{C} 3$ received trametinib monotherapy. All patients were on CombiDT at the time of Prog biopsy, except patient C7 who had ceased treatment 1 month before resection. All characterized Prog tumours were preexisting metastases that initially responded but subsequently progressed on combination therapy, except patients $\mathrm{C} 1$ and $\mathrm{C} 3$, whose Prog tumours had no clinical or radiographic response to CombiDT (Fig. 1). The median RECIST response, RECIST response rate, median PFS and OS for this cohort were 35\%, 50\%, 3.9 months and 11.8 months, respectively (Fig. 1), consistent with

\begin{tabular}{|c|c|c|c|c|c|c|c|c|c|c|c|c|c|}
\hline Patient & $\begin{array}{c}\text { Age/ } \\
\text { sex }\end{array}$ & $\begin{array}{l}\text { BRAF } \\
\text { geno } \\
\text { type }\end{array}$ & $\begin{array}{c}\text { Prior } \\
\text { MAPKi }\end{array}$ & $\begin{array}{c}\text { Daily } \\
\text { dose } \\
\text { (dab/ } \\
\text { tram) }\end{array}$ & $\begin{array}{c}\text { RECIST } \\
\text { response } \\
\text { category }\end{array}$ & $\begin{array}{c}\text { RECIST \% } \\
\text { response }\end{array}$ & $\begin{array}{c}\text { PFS } \\
\text { (months) }\end{array}$ & $\begin{array}{c}\text { OS } \\
\text { (months) }\end{array}$ & $\begin{array}{c}\text { Prog } \\
\text { no. }\end{array}$ & $\begin{array}{l}\text { Time to } \\
\text { Prog biopsy } \\
\text { (months) }\end{array}$ & Site & $\begin{array}{l}\text { Resistance } \\
\text { mechanism }\end{array}$ & $\begin{array}{c}\text { MAPK } \\
\text { activity }\end{array}$ \\
\hline $\mathrm{C} 1$ & $41 / M$ & V600E & $x$ & $300 / 1.5$ & SD & 11 & 2.6 & 3.1 & 1 & 2.8 & $\mathrm{SQ}$ & $\begin{array}{l}\text { MEK1 }{ }^{\mathrm{G} 128 \mathrm{D}} \\
\text { MEK2 }{ }^{\mathrm{C} 125 \mathrm{~S}^{\prime}}\end{array}$ & + \\
\hline $\mathrm{C} 2$ & $27 / M$ & V600K & $x$ & $300 / 2$ & SD & -14 & 3.1 & 6.8 & $\begin{array}{l}1 \\
2\end{array}$ & $\begin{array}{l}3.2 \\
4.4\end{array}$ & $\begin{array}{l}\text { SQ } \\
\text { SQ }\end{array}$ & $\begin{array}{l}\text { NRAS Q61K } \\
\text { BRAF amp }\end{array}$ & $\begin{array}{l}+ \\
+\end{array}$ \\
\hline $\mathrm{C} 3$ & $44 / M$ & V600E & $\begin{array}{l}\text { Tram- } \\
\text { PD }^{\dagger}\end{array}$ & $300 / 2$ & $\mathrm{SD}$ & -2 & 3.5 & 6.5 & 1 & 4.4 & Bowel & MEK2 ${ }^{\mathrm{C} 125 \mathrm{~S}}$ & + \\
\hline C4 & $50 / M$ & V600E & x & $150 \ddagger / 2$ & PR & -63 & 3.6 & 12.9 & 1 & 4.8 & Bowel & Unknown & + \\
\hline C5 & $46 / F$ & V600K & $x$ & $300 / 2$ & PR & -53 & 3.6 & 10.7 & 1 & 4.1 & SQ & $\begin{array}{l}\text { MEK2 } 2^{\mathrm{E} 207 \mathrm{~K}} \\
\mathrm{NRAS}^{\mathrm{G} 12 \mathrm{D}}\end{array}$ & - \\
\hline C6 & $76 / F$ & V600K & $x$ & $300 / 2$ & SD & -4 & 4.2 & 5.2 & 1 & 4.0 & $\mathrm{SQ}$ & NRASQ61K & + \\
\hline $\mathrm{C7}$ & $43 / M$ & V600E & $x$ & $150 \ddagger / 2^{\S}$ & PR & -52 & 5.3 & 13.5 & 1 & 6.4 & Peritoneum & $B R A F$ amp & + \\
\hline $\mathrm{C} 8$ & $67 / F$ & V600K & $x$ & $300 / 2$ & PR & -73 & 8.9 & $>33.5$ & 1 & 10.3 & SQ & Unknown & NA \\
\hline C9 & $66 / M$ & V600E & $x$ & $300 / 2$ & PR & -96 & 9.1 & $>15.7$ & 1 & 9.5 & SQ & $B R A F$ amp & + \\
\hline $\mathrm{C} 10$ & $55 / F$ & V600K & $\begin{array}{l}\text { Dab- } \\
\text { PR }\end{array}$ & $300 / 2$ & SD & -17 & 9.2 & $>40.0$ & 1 & 10.2 & Omentum & $B R A F$ amp & + \\
\hline
\end{tabular}


early resistance and poor clinical outcomes. Eight patients $(\mathrm{C} 1$ to C8) ceased CombiDT treatment after RECIST progression and none had evidence of tumour regression post-CombiDT cessation.

Spectrum of acquired resistance mechanisms to combined BRAF and MEK inhibition. An acquired resistance mechanism activating the MAPK pathway was identified in 9 of 11 Prog tumours (82\%; Table 1; Fig. 1); BRAF amplifications were detected in $4(36 \%), M E K 1 / 2$ mutations in $3(27 \%)$ and oncogenic NRAS mutations in 3 (27\%) Prog tumours (Figs 1 and 2). None of these alterations were identified in the matched pre-treatment samples.

Ten Prog tumours underwent gene set enrichment analysis (GSEA) of gene expression; nine showed reactivation of MAPK activity signatures, that is, MAPK pathway signalling in these progressing lesions, biopsied while the patient was receiving CombiDT, was comparable to the matched pre-treatment melanoma tumours (Table 1; Fig. 2b). Significantly, the MAPK activity in these Prog tumours was substantially higher than MAPK activity in three early during treatment (EDT) tumours, consistent with early response to CombiDT followed by subsequent disease progression. Of the nine MAPK-reactivated Prog tumours, eight had an established mechanism of resistance and one (Prog derived from patient C4) had no identified resistance driver. The Prog that did not show evidence of MAPK reactivation by GSEA (patient C5; Fig. 2b) had two identified resistance mechanisms $\left(\mathrm{MEK} 2^{\mathrm{E} 207 \mathrm{~K}}\right.$ and NRAS ${ }^{\mathrm{G} 12 \mathrm{D}}$; Supplementary Fig. 1), but both variants occurred at low frequency (13 and 15\% allelic frequency, respectively, by wholeexome sequencing; Supplementary Fig. 2), suggesting heterogeneity within the Prog metastasis. Exome sequencing revealed an $\mathrm{AKT} 3^{\mathrm{E} 17 \mathrm{~K}}$ mutation present in both the pre-treatment and Prog metastases derived from patient C5 (see below) and immunohistochemistry analyses confirmed substantial pAKT expression in the Prog tissue and in the responding EDT metastasis resected at day 9 (pre-treatment tissue was not available for this analysis; Fig. 3). Similarly, heterogeneity was seen in the resected Prog from patient $\mathrm{C} 1$. One portion of the

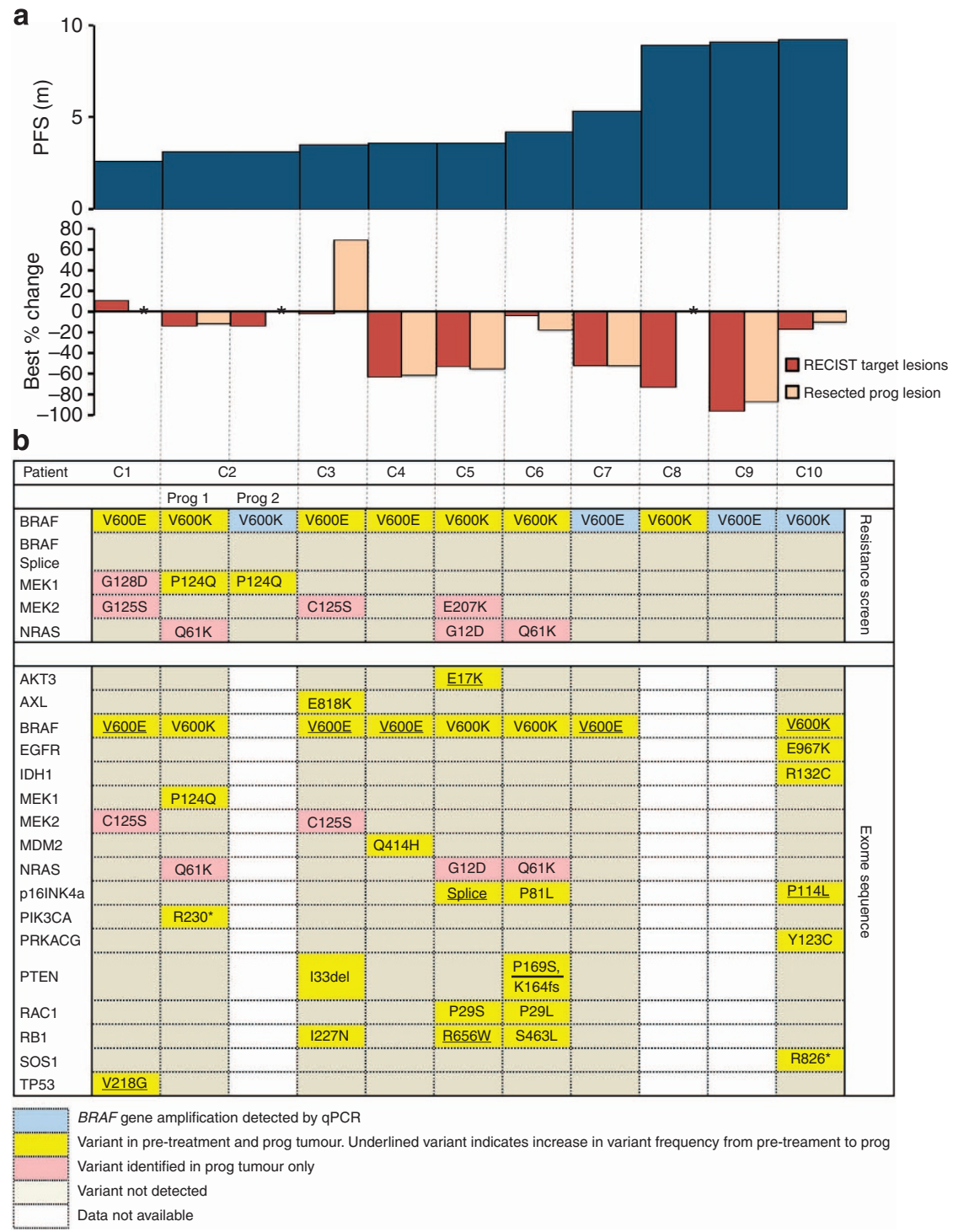

Figure 1 | Combination BRAF and MEK inhibition resistance mechanisms and clinical correlates. (a) PFS, best overall RECIST response and best Prog-specific RECIST response by mechanism of resistance to CombiDT $(n=10)$. *Best response for Progs from patients C1, C2 and C8 was not obtained. (b) Spectrum of candidate resistance variants; Prog-specific (acquired) and variants found in both pre-treatment and Prog metastases. 
a
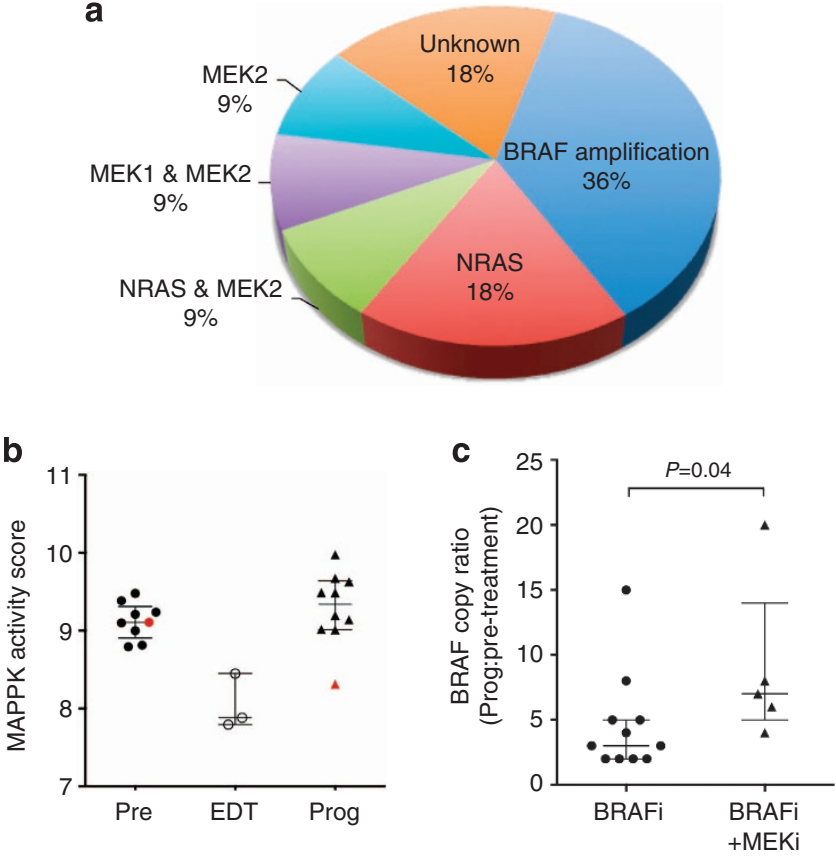

Figure 2 | MAPK signalling reactivated in combination-resistant Prog metastases. (a) Mechanisms of resistance in Prog tumours $(n=11)$. (b) Box plots showing MAPK activity (mean log2-transformed expression of MAPK activation gene transcripts) in pre-treatment (Pre; $n=9$ ) and matched Prog tumours $(n=10)$. The impact of MAPK inhibition on MAPK activity is also shown in three EDT tumours (EDT); one patient in this report (patient $\mathrm{C} 1$ ) and two previously reported BRAF inhibitor monotherapy patients ${ }^{7}$. Only the Prog sample derived from patient C5 (pre-treatment and matched Prog highlighted in red) showed significant downregulation of MAPK activity relative to the matched pre-treatment biopsy (NES $=-1.91$, false discovery rate $q<0.001$ ). MAPK activation gene set derived from ref. 18. The median values and interquartile ranges are shown. (c) Box plot showing Prog to pre-treatment ratio of BRAF gene copies in BRAF inhibitor (BRAFi)-resistant and combination-resistant (BRAFi + MEKi) Progs. Data were obtained from refs $7-9,17$. The median values and interquartile ranges are shown.

tumour screened by capillary sequencing of reverse transcription PCR (RT-PCR) products contained both the MEK1 G128D and MEK2 $2125 \mathrm{~S}$ mutations (Supplementary Fig. 1) and demonstrated MAPK reactivation. In contrast, a second portion of the same tumour was exome sequenced and contained only the $\mathrm{MEK} 2{ }^{\mathrm{C} 125 \mathrm{~S}}$ mutation (30\% allele variant frequency; Supplementary Table 1).

Selection of BRAF copy number gains and MEK2 alterations. Gain of BRAF copy number was the most common mechanism for developing resistance to CombiDT, with 4/11 Prog tumours showing amplification encompassing the $B R A F$ gene. This finding was unexpected given first the substantially lower incidence of $B R A F$ amplification in tumours progressing on BRAF inhibitor monotherapy $(18 / 140=13 \%)^{7-9}$, and second, preclinical studies showing that overexpression of BRAF in sensitive melanoma cells confers resistance to MEK inhibitor monotherapy, but they remain sensitive to combined BRAF and MEK inhibition ${ }^{10}$. We therefore examined the extent of $B R A F$ amplification in several cohorts $^{7-9,17}$, and, although the numbers of tumours available for analyses were small, we found a significant increase in the BRAF Prog to pre-treatment copy number ratio in CombiDT-resistant

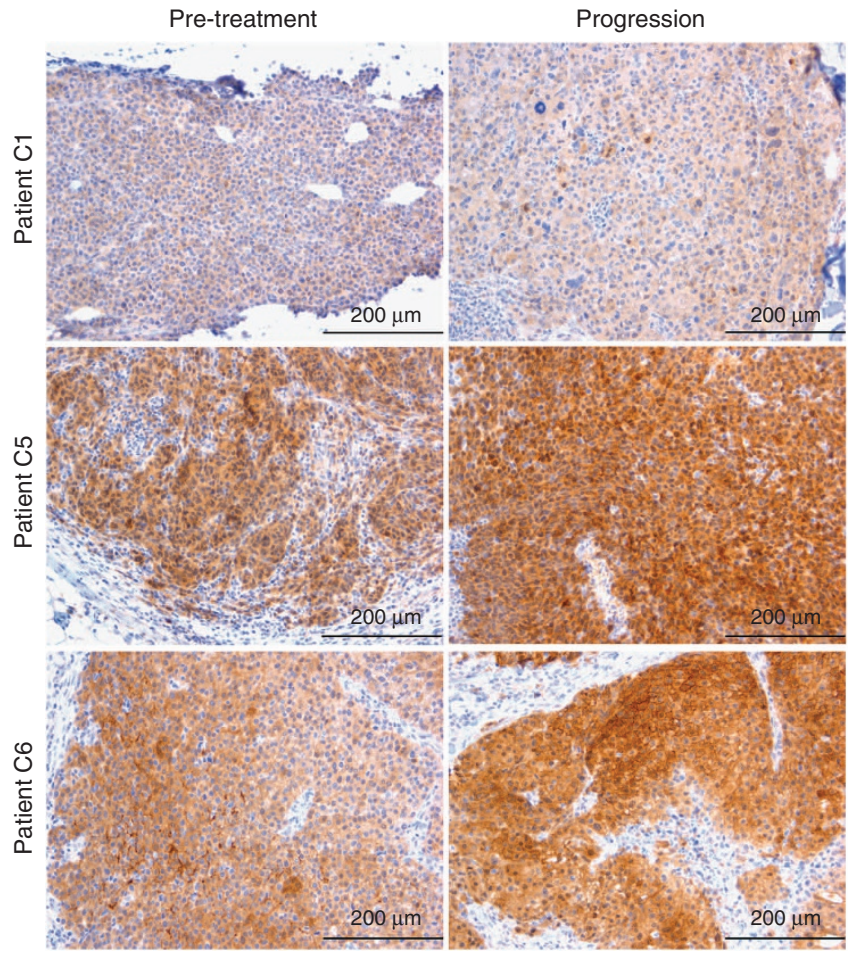

Figure 3 | Expression of pAKT in matched pre- or early-treatment and Prog tumours derived from patients C1, C5 and C6. High levels of phosphorylated AKT in the matched pre-treatment and Prog tumour samples derived from patients $\mathrm{C} 5$ and $\mathrm{C} 6$. AKT3 $3^{\mathrm{E} 17 \mathrm{~K}}$ and RAC1 ${ }^{\mathrm{P} 29 \mathrm{~S}}$ mutations were identified in pre-treatment and Prog tumours derived from patient $\mathrm{C5}$, and RAC1 ${ }^{\mathrm{P} 129 \mathrm{~L}}$ and PTEN ${ }^{\mathrm{K} 164 \mathrm{fs}}$ mutations were identified in the pre-treatment and Prog tumours derived from patient C6. In contrast, AKT phosphorylation was low in the pre-treatment and Prog tumours derived from patient $\mathrm{C}$. These tumours had no detectable oncogenic PI3K-AKT pathway mutations. For patient C5, the EDT tumour (taken at day 9 of a CombiDT-responding lesion) is shown in place of a pre-treatment tumour.

tumours compared with tumours progressing on BRAF inhibitor monotherapy ( $P=0.04$; Fig. $2 \mathrm{c})$.

BRAF-splice variants have been identified as a mechanism of resistance to BRAF inhibitor monotherapy ${ }^{7,8,12}$. In contrast, no BRAF-splice isoforms were detected in the 11 CombiDT-resistant melanomas in this cohort. Analysis of patient-derived and in vitro-generated dabrafenib-resistant melanoma cell lines expressing endogenous BRAF-splice variants lacking exons 2-8, 2-10 and 4-10 (ref. 14) confirmed that these cells were resistant to dabrafenib monotherapy but retained sensitivity to singleagent trametinib (Fig. 4a).

These apparent differences in $B R A F$ gene alterations between tumours resistant to mono- or combination MAPK inhibition suggested that BRAF genetic alterations might differ in their activation of downstream MAPK signalling. We therefore examined MAPK signalling using an established gene transcription signature, indicative of persistent MAPK activation ${ }^{18}$. A comparison between five BRAF-amplified Progs (including one dabrafenib-resistant BRAF-amplified Prog tumour ${ }^{7}$ ) and seven previously described BRAF-splice variant Prog tumours ${ }^{7}$ confirmed significantly elevated MAPK signalling in the BRAFamplified class (GSEA normalized enrichment score $=2.07$, false discovery rate $q=0.00, P=0.00$; Fig. $4 \mathrm{~b}$ ).

Mutations affecting MEK1 and MEK2 have each been identified in $8 / 154(5 \%)$ and $5 / 154$ (3\%) of melanomas progressing on BRAF inhibitor monotherapy, respectively ${ }^{7-9}$. In 


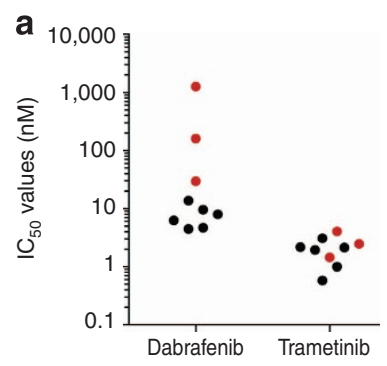

b

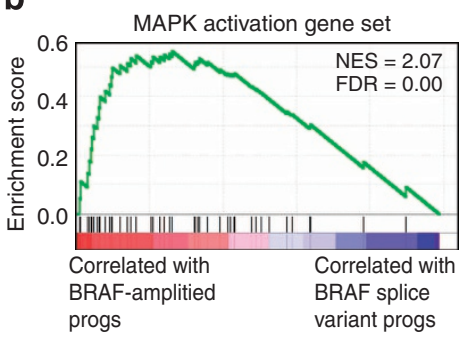

Figure 4 | BRAF-splice variants confer diminished BRAF inhibitor sensitivity, but remain sensitive to MEK inhibition. (a) $I C_{50}$ values of dabrafenib and trametinib in a panel of BRAF-mutant melanoma cell lines. The SMU027 and WMD009 patient-derived cells and BR4 in vitro generated dabrafenib-resistant cell lines that express BRAF-splice isoforms (BRAF ${ }^{\mathrm{e} 2-8 \Delta}, \mathrm{BRAF}^{\mathrm{e} 2 \mathrm{2}-10 \Delta}, \mathrm{BRAF}^{\mathrm{e} \times 4-10 \Delta}$; ref. 14) and are highlighted in red. Other melanoma cells include NM176, MM200, M238, M249, SKMel28 and NM182 (black). (b) GSEA shows enrichment of MAPK activation gene set in Prog tumours with BRAF copy number gains $(n=5)$ versus Prog tumours with BRAF-splice isoforms $(n=7)$. MAPK activation gene set derived from ref. 18. The false discovery rate (FDR) statistic and normalized enrichment score (NES) are indicated.

comparison, we found that MEK2 alterations were predominant over MEK1 genetic alterations in CombiDT-resistant Progs (3/ $11=27 \%$ ). The relative frequency of acquired MEK2 mutations was significantly increased in melanomas progressing on combination dabrafenib/trametinib (4/16; this report and ref. 16) compared with melanomas progressing on BRAF inhibitor monotherapy (5/154; Fisher's exact test; $P=0.01$ ). Functional analyses of MEK1 and MEK2 mutant proteins with mutations in equivalent residues-MEK1 ${ }^{\mathrm{C} 121 \mathrm{~S}}$ versus MEK2 ${ }^{\mathrm{C} 125 \mathrm{~S}}$-confirmed that, although both mutant MEK proteins displayed elevated ERK kinase activity, relative to the wild-type MEK protein (Supplementary Fig. 3), only expression of the MEK2 ${ }^{\mathrm{Cl}} 25 \mathrm{~S}$ mutant (found in two combination-resistant Progs; patients $\mathrm{C} 1$ and C3) was associated with significant levels of phosphorylated retinoblastoma protein (a marker of cell proliferation), phosphorylated ERK and colony growth in the presence of combined dabrafenib and trametinib (Fig. 5).

Exome analysis and mutations affecting MAPK, PI3K and other pathways. We also sequenced the exomes of eight matched pre-treatment and Prog melanoma pairs, including patient $\mathrm{C} 4$ who had unknown mechanisms of resistance and MAPK reactivation (Table 1; Fig. 1). All tumours retained the $\mathrm{BRAF}^{\mathrm{V} 600}$ mutation, and mutant BRAF allele frequency gains from pre-treatment to Prog tumour were significant in four patients (C1, C4, C7 and C10), two of which (patients C7 and C10) showed substantial BRAF gene amplification (mutant and wild type) by quantitative PCR (qPCR) and the other two showing diploid levels of the BRAF gene by qPCR. The exome-sequencing data were consistent with our RT-PCR Sanger sequencing resistance data, except for patient $\mathrm{C} 1$ who displayed heterogeneity of the MEK $1^{\mathrm{G} 128 \mathrm{D}}$ mutation; this mutation was not present in the portion of the Prog tumour that was exome sequenced. The MEK2 $2^{\mathrm{E} 207 \mathrm{~K}}$ mutation identified in the Prog from patient C5 was evident in the next generation sequence data (Supplementary Fig. 2), but was not called due to low-quality value (Supplementary Fig. 2).

To identify alterations that may diminish response to MAPK inhibition, we cross-referenced recurrently mutated genes in the COSMIC database, genes in the PI3K (KEGG ID:hsa04151) and MAPK (KEGG ID:hsa04010) pathways and genes purported
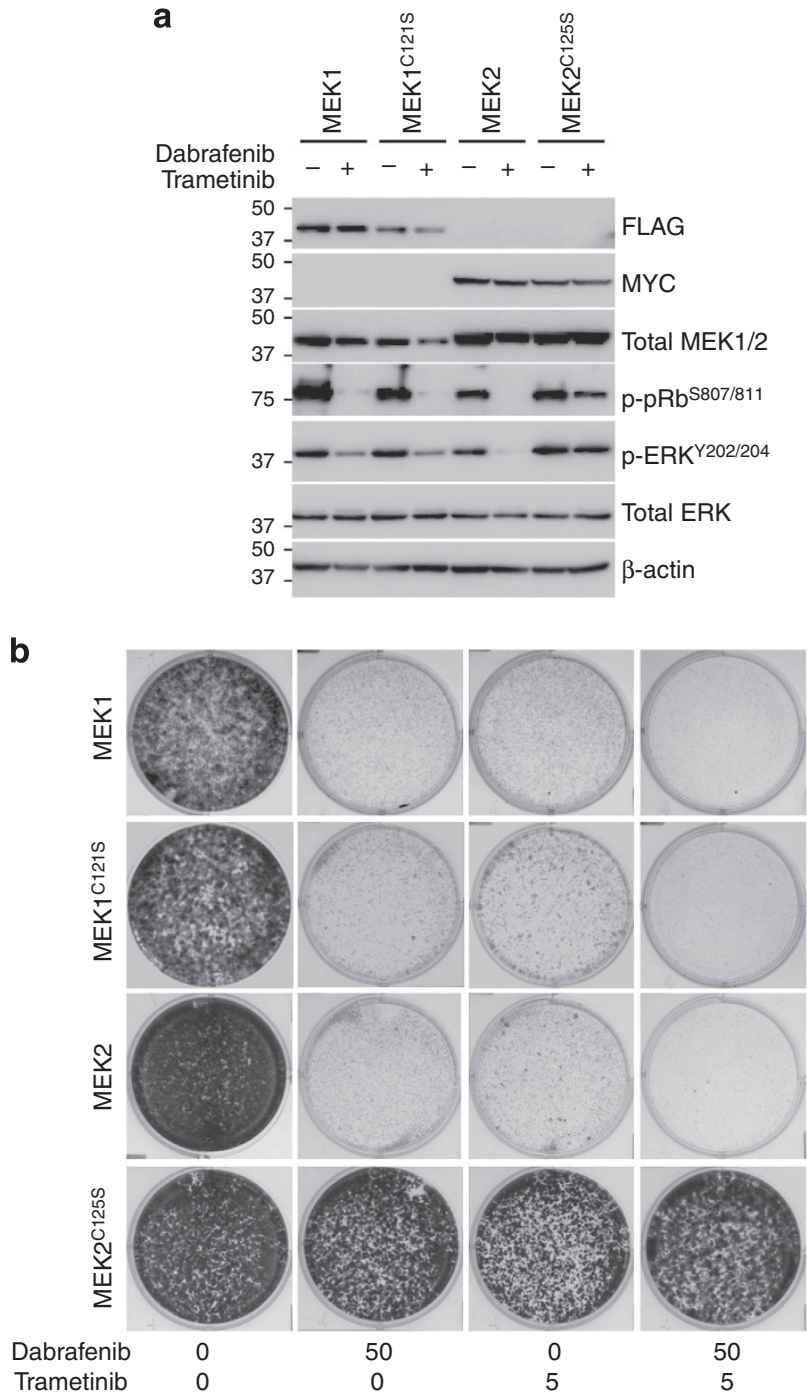

Figure 5 | MEK2 ${ }^{\mathrm{C} 125 \mathrm{~S}}$, but not the equivalent MEK1 ${ }^{\mathrm{C} 121 S}$ variant, confers robust resistance to dabrafenib and trametinib. (a) SKMel28 melanoma cells were stably transduced with the indicated MEK constructs. Cell lysates were analysed for the indicated proteins $48 \mathrm{~h}$ after incubation with dabrafenib (50 $\mathrm{nM})$ and trametinib ( $5 \mathrm{nM})$. Ectopically expressed MEK1 and MEK2 proteins were tagged with the FLAG and MYC epitopes, respectively. (b) Transduced SKMel28 cells were seeded at low density and $24 \mathrm{~h}$ after seeding were treated with the indicated concentrations of dabrafenib and trametinib every $72-96 \mathrm{~h}$. Colonies were stained with crystal violet 10 days post transduction. Photographs are representative of at least two independent transduction experiments.

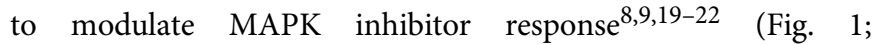
Supplementary Table 1). Novel Prog-specific mutations were uncommon, although gains in mutant allele frequency from pretreatment to Prog were seen in most patients (Fig. 1; Supplementary Table 1).

Five patients (patients $\mathrm{C} 2, \mathrm{C} 3, \mathrm{C} 5, \mathrm{C} 6$ and C10) had tumours with alterations within the PI3K-AKT-mTOR pathway. The activating $\mathrm{AKT}^{\mathrm{E}}{ }^{\mathrm{E} 7 \mathrm{~K}}$ mutation ${ }^{23}$ was identified in matched pretreatment and Prog biopsies derived from patient C5 and the PTEN $^{\mathrm{I3} 3 \mathrm{del}}$ (ref. 24) and PTEN ${ }^{\mathrm{K} 164 \mathrm{fs}}$ (COSMIC) alterations were found in melanomas derived from patients $\mathrm{C} 3$ and C6, respectively. Uncharacterized mutations affecting AXL (E818K), PTEN (P169S) and SOS1 (R826*) were identified in matched pretreatment and Prog biopsies derived from patients C3, C6 and 
C10, respectively. Oncogenic mutations activating the GTPase RAC1 (P29S and P29L) ${ }^{25-27}$ were found in matched pretreatment and Prog melanomas derived from patients C5 and C6.

Six patients (patients C1, C3, C4, C5, C6 and C10) had tumours with alterations potentially affecting key proteins of the cell cycle. Melanoma-associated inactivating mutations affecting p16 ${ }^{\mathrm{INK} 4 \mathrm{a}}$ $(\mathrm{c} 150+1 \mathrm{G}>\mathrm{A}$ splice variant, P114L and P81L (refs 28,29), patients C5, C6 and C10) and previously unidentified missense RB1 variants (I227N, patient C3; S463L, patient C6; and R656W, patient C5) were also found in the pre-treatment and Prog tumours. Alterations potentially affecting the p53 pathway, including a novel MDM2 missense variant (Q414H, patient $\mathrm{C} 4)$ and the inactivating $\mathrm{p} 53^{\mathrm{V} 218 \mathrm{G}}$ mutation (patient $\left.\mathrm{C} 1\right)^{30}$, were also found in pre-treatment and Prog-matching tumours.

The glioma-associated neomorphic mutation in the isocitrate dehydrogenase 1 gene (IDH1 ${ }^{\mathrm{R} 132 \mathrm{C}}$; refs 31,32 ) was identified in the baseline and progressing melanomas derived from patient C10. Patient C7 had no PI3K-AKT alterations identified by exome sequencing in pre-treatment and Prog tumours, yet displayed early resistance with BRAF amplification as the Progspecific mechanism of resistance. Other alterations identified in both pre-treatment and Prog tumours included novel variants affecting MET, EGFR, SOS2 and RAC2 (Supplementary Table 1).

We also confirmed that PI3K-AKT signalling, as evidenced by AKT phosphorylation, correlated with $\mathrm{AKT} 3^{\mathrm{E} 17 \mathrm{~K}} / \mathrm{RAC1}^{\mathrm{P} 29 \mathrm{~S}}$ mutations and RAC1 $1^{\mathrm{P} 29 \mathrm{~L}} / \mathrm{PTEN}^{\mathrm{K} 164 \mathrm{fs}}$ mutations in matching tumours derived from patients C5 and C6, respectively (Fig. 3), indicating that $\mathrm{AKT}$ activation was present before, during and on combiDT therapy progression. In contrast, AKT phosphorylation was low in the tumours derived from patient $\mathrm{C} 1$, who had no detectable alterations activating the PI3K-AKT pathway. Thus, despite the potential importance of this parallel signalling pathway for escape from MAPK inhibition, we did not identify any Prog-specific exome alterations affecting the PI3K-AKTmTOR network in these CombiDT-resistant tumours.

\section{Discussion}

This study describes the largest set of metastatic melanoma patients analysed for resistance mechanisms to combined treatment with BRAF and MEK inhibitors. Most of these patients responded poorly to CombiDT (median tumour reduction was $35 \%$, RECIST response rate $50 \%$ ) and progressed early (median PFS 3.6 months) when compared with those previously reported ${ }^{1}$. These patients represent a subgroup with early resistance and are in particular urgent need of new, more effective treatment strategies. Nearly all Prog tumours $(9 / 11,82 \%)$ had an identified mechanism of resistance that reactivated MAPK signalling and was not detected in the matched pre-treatment biopsy. This compares with our previous report on resistance to BRAF inhibitor monotherapy in which only $50 \%$ of BRAF inhibitor Prog tumours had an identified resistance mechanism and early resistance was associated with continued MAPK inhibition, rather than reactivation ${ }^{7}$.

Reactivation of the MAPK pathway occurred primarily via alterations affecting the drug targets (that is, BRAF copy number gains and MEK2 mutations). Although $B R A F$ gene amplification and MEK mutations were also identified in melanomas resistant to BRAF inhibitor monotherapy ${ }^{7-10,13}$, our data show that the upregulation of MAPK signalling was substantially greater in melanomas resistant to CombiDT compared with melanomas resistant to BRAF inhibitor monotherapy. This was most evident when comparing ratios of Prog/pre-treatment BRAF copy number. BRAF amplifications and MAPK output were significantly elevated in the CombiDT-resistant tumours. The significant role of MAPK signalling output in conferring resistance was also evident when analysing BRAF-splice isoforms. The level of MAPK signalling driven by these truncated BRAF proteins was lower relative to that seen with BRAF amplifications, and insufficient to confer trametinib resistance. The recent identification of a BRAF-splice isoform lacking exons 2-10 in a single CombiDT-resistant melanoma suggests that additional genetic alterations may have contributed to drug resistance in that sample ${ }^{16}$.

Despite the very high level of mutations in melanomas ${ }^{19,22,33}$, it is salutary that these tumours retain such high dependency on MAPK activity. The predominance of MAPK reactivation in tumours progressing on CombiDT is unexpected and carries important implications for future clinical trial design strategies in these patients. The abundance of $B R A F$ amplifications is of particular note as ERK inhibitors are active in the context of BRAF copy number gains ${ }^{34}$, including in models resistant to combined BRAF and MEK inhibition ${ }^{14}$. Furthermore, these data suggest that, in unselected patients with CombiDT failure, drug combinations involving PI3K/AKT inhibition (for example, NCT 01902173; ref. 35) may prove less successful than additional therapies inhibiting MAPK at multiple nodes, such as those targeting MEK, ERK and possibly CDK4 (NCT01777776, NCT01543698).

Although trametinib shows similar inhibitory activity towards the MEK1 and MEK2 proteins in in vitro ERK kinase assays ${ }^{36}$, we observed a preponderance of MEK2 mutations rather than MEK1 mutations in CombiDT-resistant Progs. The fact that the $\mathrm{MEK} 2^{\mathrm{C} 125 \mathrm{~S}}$ mutation and not the homologous MEK1 ${ }^{\mathrm{C} 121 \mathrm{~S}}$ conferred robust resistance to combination therapy in our preclinical models highlights the functional differences between these highly homologous kinases. Previous reports indicating that MEK2, rather than MEK1, can escape inhibition by the allosteric MEK inhibitor AZD6244 (ref. 37) and that MEK2 is the more potent ERK activator ${ }^{38,39}$ are consistent with our data and recent reports of MEK2 mutations in CombiDT-resistant melanomas $^{16,17}$. Current data suggest that ERK activity is gradually restored in the presence of BRAF and MEK inhibitors via receptor tyrosine kinase reprogramming driven by the loss of suppressive feedback regulation ${ }^{11,37,40}$. The reactivation of ERK promotes the phosphorylation of MEK1 at the negative regulatory Thr292 residue and this facilitates MEK1 inactivation via the dephosphorylation of the activating serine residues ${ }^{41,42}$. In contrast, MEK2, which lacks the threonine-negative regulatory site, remains phosphorylated at the activation loop serines in the presence of ERK activity. Activated, phosphorylated MEK proteins also have a significantly lower affinity for trametinib ${ }^{37}$. We are currently exploring the impact of these phosphorylation events on the activity of the MEK1 ${ }^{\mathrm{C} 121 \mathrm{~S}}$ and MEK2 ${ }^{\mathrm{C} 125 \mathrm{~S}}$ proteins.

Despite the potential importance of the PI3K-PTEN-AKT signalling pathway for escape from MAPK inhibition, we did not identify Prog-specific genetic alterations affecting this pathway. Instead, many tumours carried pre-existing genetic variants, including RAC1, PTEN and AKT3 mutations that activate the PI3K-PTEN-AKT cascade. Consequently, although the sole activation of PI3K activity may not be sufficient to confer CombiDT resistance, we predict that PI3K activity modulates tumour responses to MAPK inhibitors and contributes to early resistance, and that combination MAPK and PI3K/AKT inhibition may be best administered upfront to delay the emergence of resistance ${ }^{35}$. Preclinical data have confirmed that PI3K activation (via NRAS mutations and receptor tyrosine kinase activation) diminished melanoma cell responses to combination BRAF and MEK inhibitors ${ }^{14}$. The hotspot RAC $^{\mathrm{P} 29 \mathrm{~S}}$ mutation also confers resistance to RAF and MEK inhibitors ${ }^{26}$. Mutations affecting p16 ${ }^{\text {INK4a }}$ were also found at baseline and inactivation of this tumour suppressor was associated with a shorter PFS in patients 
treated with dabrafenib ${ }^{43}$. Loss of $\mathrm{p} 16^{\text {INK4a }}$, PTEN and $\mathrm{pRb}$ also contributed to intrinsic BRAF inhibitor and MEK inhibitor resistance in vitro ${ }^{44,45}$.

This cohort was distinguished by the presence of $\mathrm{BRAF}^{\mathrm{V} 600 \mathrm{~K}}$ mutant melanoma in $50 \%$ of patients, a mutation that is present in 6-30\% of melanoma tumours ${ }^{46,47}$. Patients with $\mathrm{BRAF}^{\mathrm{V} 600 \mathrm{~K}}$ and the more common $\mathrm{BRAF}^{\mathrm{V} 600 \mathrm{E}}$-mutant melanoma show significant responses to BRAF inhibitor and combination therapy ${ }^{1,48}$, and, although some studies suggest that dabrafenib may be less active in $\mathrm{BRAF}^{\mathrm{V} 600 \mathrm{~K}}$-mutant melanoma compared with $\mathrm{BRAF}^{\mathrm{V} 600 \mathrm{E}}$-mutant disease ${ }^{48-50}$, the response rate, median PFS and OS in vemurafenib-treated patients were similar between the two BRAF genotypes ${ }^{51}$.

This study suggests that the genetic effectors mediating acquired resistance to combination BRAF and MEK inhibitors may not be as diverse as those seen with BRAF inhibitor monotherapy. Perhaps, this is not surprising as the simultaneous inhibition of BRAF and MEK1/2 may negate many MAPK resistance effectors signalling above MEK. Surprisingly, MAPKindependent mechanisms of resistance were not acquired at a higher frequency than that in single BRAF inhibitor resistance, suggesting that patients progressing on combination dabrafenib/ trametinib therapy may be responsive to drug combinations targeting multiple members of the MAPK pathway and its downstream targets including ERK and CDK4. Durable responses in BRAF-mutant melanoma patients may require combination first-line therapy that selectively inhibits multiple proliferative and survival pathways.

\begin{abstract}
Methods
Patients, treatment and assessments. Patients included in this study had $\mathrm{BRAF}^{\mathrm{V} 600}$-mutant metastatic melanoma, had not received prior immunotherapy and were treated with dabrafenib plus trametinib (CombiDT) as part of phase I/II or III clinical trials ${ }^{1}$ (NCT01584648, NCT01597908). The majority of patients received maximal recommended daily doses of dabrafenib $(300 \mathrm{mg})$ plus trametinib ( $2 \mathrm{mg}$; Table 1). Nine of 10 patients had a pre-treatment biopsy and all patients had at least one progressing melanoma metastasis (Prog) resected. Initial Prog lesions were resected within 6 weeks of RECIST-determined disease progression.

Subsequent additional Prog biopsies were procured from patients remaining on CombiDT treatment beyond progression.

Clinical outcome was assessed using PFS and OS from commencement of CombiDT and RECIST-defined response ${ }^{52}$. Informed consent was obtained for each patient under approved Human Research Ethics Committee protocols.
\end{abstract}

Melanoma tissue samples. Fresh melanoma samples were macrodissected by a pathologist and enriched tumour portions snap frozen. Frozen tumour sections were cut, stained with hematoxylin and eosin and scored for the following parameters: \% non-tumour cells, \% necrosis, degree of pigmentation, predominant cell shape and cell size of the most cellular portion of tumour ${ }^{53}$. Tumour foci were carefully macrodissected utilizing the marked frozen section as a guide to meet minimum criteria for tumour cell content $(>80 \%)$ and amount of necrosis $(<30 \%)$. High-percentage tumour content was verified by examining postmacrodissection frozen section slides. Total RNA was extracted from 10 to $20 \mathrm{mg}$ of tumour. Tissue samples were homogenized using a high-speed agitation Polytron blender (Kinematica, Luzern, Switzerland) in the presence of Trizol (Life Technologies, Carlsbad, CA, USA). Following homogenization, chloroform was added and the sample centrifuged. The upper phase was removed and mixed with $70 \%$ ethanol. The RNA was then isolated and purified with an RNeasy purification kit with DNase I digestion on the column (Qiagen, Valencia, CA, USA). The quality of the RNA preparations was assessed using a Agilent 2100 Bioanalyser (Agilent Technologies, Palo Alto, CA, USA). The extracted total RNA was of high quality (RNA integrity number: $8-10$ ).

\section{Analysis of resistance mechanisms and gene expression analysis. The} expression of BRAF-splice variants, the complete coding sequence of MEK1, MEK2 and NRAS complementary DNAs and the $5^{\prime}$ half of the AKT1 cDNA were examined using an RT-PCR screen ${ }^{7}$. Reverse transcription reactions were performed using the Superscript III First-Strand Synthesis kit (Invitrogen) with the oligo dT primer. The MEK1, MEK2, NRAS and AKT1 gene products were each amplified from cDNA using Taq polymerase (Fisher Biotech, Wembley, WA, Australia) and $B R A F$ cDNA was amplified with Pfx polymerase (Life

Technologies). Amplification and sequencing primers are listed in Supplementary Table 2. PCR products were purified using QIAquick PCR purification kit (Qiagen,
Limburg, Netherlands) followed by Sanger sequencing on the 3730xl DNA Analyser (AGRF, Westmead, NSW, Australia). BRAF relative copy number was determined by qPCR using the Corbett Rotor-Gene 6000 (ref. 54).

Gene expression analysis was performed on matched pre-treatment $(n=9)$ and Prog $(n=10)$ biopsies derived from nine patients, using the Sentrix HumanHT12 v.4.0 Expression BeadChip (Illumina, San Diego, CA, USA). Complementary RNA amplification and labelling with biotin were performed using the Illumina TotalPrep RNA amplification kit (Ambion, Austin, TX, USA) according to the manufacturer's instructions starting with $250 \mathrm{ng}$ total $\mathrm{RNA}^{7}$. Three responding (that is, shrinking) melanoma samples taken early during BRAF inhibitor monotherapy $(n=2)$ or combination BRAF and MEK inhibitor therapy $(n=1$; EDT; 2-7 days after therapy initiation) were also included in these gene expression analyses. Reproducibility of gene expression data was confirmed by including technical replicates, in which RNA was independently amplified and hybridized on different arrays and on separate days. Clustering using GenomeStudio (Illumina) confirmed that each replicate showed $<1.2 \%$ variation. The microarray platform and data have been submitted to the Gene Expression Omnibus public database at the National Center for Biotechnology Information, following the minimum information about microarray gene experiment guidelines. The accession code is GSE61992.

Exome sequencing. Exome sequencing was performed on patient-matched Pre and Prog melanoma tissues. Exonic DNA was enriched using the Illumina TrueSeq technology, targeting $62 \mathrm{Mb}$ encompassing protein-coding regions (Supplementary Table 3), and sequenced on an Illumina HiSeq2000. We aligned the read pairs to the reference human genome (hg19) using Burrows-Wheeler Aligner (BWA) ${ }^{55}$, removed duplicates with Picard and applied GATK indel realignment and base quality recalibration ${ }^{56}$. Single-nucleotide variants and small insertion/deletions (INDELS) were detected by SAMTools ${ }^{57}$. To generate a list of high-quality variants, we removed low-coverage variants (single-nucleotide polymorphism quality $<30$ read $<30$ ) and eliminated variants annotated as common polymorphisms or in the 1000 Genomes Project. We used Ingenuity Variant Analysis (http:// www.ingenuity.com) to compare the remaining mutations between the pretreatment and Prog samples from each patient to detect oncogenic variants and Prog-specific cancer-driver mutations. When a somatic mutation was present in both the pre-treatment and Prog samples for a given patient, Fisher's exact test was used to determine whether the difference in allele frequency was statistically significant.

Cell culture and constructs. Short-term cultures were established from a subset of patients by mechanically processing tissue in Dulbecco's Modified Eagle Medium (DMEM) $/ 10 \%$ fetal calf serum (FCS) containing $100 \mathrm{U} \mathrm{ml}^{-1}$ penicillin and $100 \mu \mathrm{g} \mathrm{ml}^{-1}$ streptomycin (Gibco BRL). The clarified cell suspension was maintained in DMEM/10\% FCS containing penicillin and streptomycin (Gibco). Contaminating fibroblasts were removed by treating cultures with $100 \mu \mathrm{g} \mathrm{ml}^{-1} \mathrm{G} 418$ (Gibco) as required ${ }^{58}$. Cells were grown in DMEM with $10 \%$ FCS and glutamine (Gibco BRL) and cultured in a $37^{\circ} \mathrm{C}$ incubator with $5 \% \mathrm{CO}_{2}$. Stocks of dabrafenib (supplied by Active Biochem, Maplewood, NJ, USA) and trametinib (Selleck Chemicals, Houston, TX, USA) were made in dimethyl sulfoxide. Cell authentication was confirmed using the StemElite ID system from Promega. Lentiviruses were produced in HEK293T cells using expression vectors encased in viral capsid encoded by three packaging plasmids as described earlier ${ }^{59,60}$. Cells were infected using a multiplicity of infection of 1-5 to provide an efficiency of infection above $90 \%$. Wild-type and mutant FLAG-tagged MEK1 and MYC-tagged MEK2 constructs were each cloned into the $p C D H$-CuO-MCS-EF1-CymR-PURO and plenti6.3/T0/V5-DEST lentiviral vectors, respectively.

Clonogenic and pharmacological growth assays. Approximately $96 \mathrm{~h}$ posttransduction, melanoma cells were seeded at 25,000 cells per well onto six-well culture plates and allowed to grow for $24 \mathrm{~h}$ followed by the desired treatment. Cells were then washed twice with ice-cold PBS and fixed with ice-cold methanol for $1 \mathrm{~min}$. After aspirating methanol from plates, $0.1 \%$ crystal violet (Sciencelab.com, TX, USA) solution was added and the plate was incubated at room temperature for $5 \mathrm{~min}$. Distilled water was used to rinse the plate. Pharmacological growth inhibition assays were performed using $1-2 \times 10^{3}$ cells per well in 96-well plates and $24 \mathrm{~h}$ after seeding, serial dilutions of each inhibitor were added to cells. Cells were incubated for an additional $72 \mathrm{~h}$ and cell viability was measured using the Cell proliferation Aqueous MTS assay (Promega) on a VICTOR ${ }^{2}$ Multilabel counter $\left.{ }^{(P e r k i n E l m e r}\right)^{58}$.

Western blotting and immunohistochemistry. Total cellular proteins were extracted at $4{ }^{\circ} \mathrm{C}$ using radioimmunoprecipitation assay lysis buffer containing protease inhibitors and phosphatase inhibitors (Roche). Proteins $(40 \mu \mathrm{g})$ were resolved on $12 \%$ SDS-polyacrylamide gels and transferred to Immobilon-P membranes (Millipore). Western blots were probed with the following antibodies: total ERK (1:2000; 137F5; Cell Signaling, Danvers, MA, USA), phosphorylated ERK (1:2000; E-4; Santa Cruz, Santa Cruz, CA, USA), MYC (1:1000; A-14; Santa Cruz), FLAG (1:1000; Sigma-Aldrich, St Louis, MO, USA), MEK1/2 (1:2000; L38C12, Cell 
Signaling), phosphorylated pRb (1:500; Cell Signaling) and $\beta$-actin (1:6000; AC-74; Sigma-Aldrich).

Immunohistochemical staining of selected matched formalin-fixed, paraffinembedded melanoma specimens using phosphorylated AKT (1:75; CS-3787; Cell Signaling) was performed on a Dako Autostainer Plus (Dako, Glostrup, Denmark), utilizing the EnVision FLEX, High pH visualization system (K8024, Dako) according to the manufacturer's protocol. Sections $(4 \mu \mathrm{m})$ were baked at $58^{\circ} \mathrm{C}$ for $60 \mathrm{~min}$, then underwent deparaffinization and antigen retrieval using the heatinduced epitope retrieval method with Envision Flex Target Retrieval Solution (High $\mathrm{pH}$ ) in a PT link unit (Dako). Antibody detection was performed using the Dako Envision Flex HRP (Dako) and visualized using 3,3'-diaminobenzidine (Dako), and the slides were counterstained with Harris's hematoxylin? ${ }^{7}$.

Statistical analysis. The Kaplan-Meier method was used to calculate median OS and PFS from the date of commencement on combination therapy, together with the Cox-Mantel log-rank test to calculate statistical significance where appropriate. Confidence intervals for proportions were calculated without continuity correction. The Mann-Whitney $U$-test was used to compare medians for nonparametric groups.

\section{References}

1. Flaherty, K. T. et al. Combined BRAF and MEK inhibition in melanoma with BRAF V600 mutations. New Engl. J. Med. 367, 1694-1703 (2012).

2. Long, G. V. et al. Combined BRAF and MEK inhibition versus BRAF inhibition alone in melanoma. New Engl. J. Med. doi:10.1056/NEJM.a1406037 (2014).

3. Robert, C. et al. COMBI-v: A randomised, open-label, phase III study comparing the combination of dabrafenib (D) and trametinib (T) to vemurafenib $(\mathrm{V})$ as first-line therapy in patients (pts) with unresectable or metastatic BRAF V600E/K mutation-positive cutaneous melanoma. Ann. Oncol. 25, 1-41 (2014).

4. Larkin, J. et al. Combined Vemurafenib and Cobimetinib in BRAF-mutated melanoma. New Engl. J. Med. doi:10.1056/NEJM.a1408868 (2014)

5. Kefford, R. et al. Preliminary results from a phase Ib/II, open-label, doseescalation study of the oral BRAF inhibitor LGX818 in combination with the oral MEK1/2 inhibitor MEK162 in BRAF V600-dependent advanced solid tumors. ASCO Meet. 31, 9029 (2013).

6. Menzies, A. M. et al. Inter- and intra-patient heterogeneity of response and progression to targeted therapy in metastatic melanoma. PLoS ONE 9, e85004 (2014).

7. Rizos, H. et al. BRAF inhibitor resistance mechanisms in metastatic melanoma: spectrum and clinical impact. Clin. Cancer Res. 20, 1965-1977 (2014).

8. Shi, H. et al. Acquired resistance and clonal evolution in melanoma during BRAF inhibitor therapy. Cancer Discov. 4, 80-93 (2014).

9. Van Allen, E. M. et al. The genetic landscape of clinical resistance to RAF inhibition in metastatic melanoma. Cancer Discov. 4, 94-109 (2014).

10. Shi, H. et al. Melanoma whole-exome sequencing identifies (V600E)B-RAF amplification-mediated acquired B-RAF inhibitor resistance. Nat. Commun. 3, 724 (2012).

11. Nazarian, R. et al. Melanomas acquire resistance to B-RAF(V600E) inhibition by RTK or N-RAS upregulation. Nature 468, 973-977 (2010).

12. Poulikakos, P. I. et al. RAF inhibitor resistance is mediated by dimerization of aberrantly spliced BRAF(V600E). Nature 480, 387-390 (2011).

13. Wagle, N. et al. Dissecting therapeutic resistance to RAF inhibition in melanoma by tumor genomic profiling. J. Clin. Oncol. 29, 3085-3096 (2011).

14. Carlino, M. S. et al. Differential activity of MEK and ERK inhibitors in BRAF inhibitor resistant melanoma. Mol. Oncol. 15, 544-554 (2014).

15. Gowrishankar, K. et al. Acquired resistance to BRAF inhibition can confer cross-resistance to combined BRAF/MEK inhibition. J. Invest. Dermatol. 132, 1850-1859 (2012).

16. Wagle, N. et al. MAP kinase pathway alterations in BRAF-mutant melanoma patients with acquired resistance to combined RAF/MEK inhibition. Cancer Discov. 4, 61-68 (2014).

17. Villanueva, J. et al. Concurrent MEK2 mutation and BRAF amplification confer resistance to BRAF and MEK inhibitors in melanoma. Cell Rep. 4, 1090-1099 (2013).

18. Pratilas, C. A. et al. V600E)BRAF is associated with disabled feedback inhibition of RAF-MEK signaling and elevated transcriptional output of the pathway. Proc. Natl Acad. Sci. USA 106, 4519-4524 (2009).

19. Hodis, E. et al. A landscape of driver mutations in melanoma. Cell 150, 251-263 (2012).

20. Johannessen, C. M. et al. A melanocyte lineage program confers resistance to MAP kinase pathway inhibition. Nature 504, 138-142 (2013).

21. Whittaker, S. R. et al. A genome-scale RNA interference screen implicates NF1 loss in resistance to RAF inhibition. Cancer Discov. 3, 350-362 (2013).

22. Mar, V. J. et al. BRAF/NRAS wild-type melanomas have a high mutation load correlating with histologic and molecular signatures of UV damage. Clin. Cancer Res. 19, 4589-4598 (2013).
23. Davies, M. A. et al. A novel AKT3 mutation in melanoma tumours and cell lines. Br. J. Cancer 99, 1265-1268 (2008).

24. Nelen, M. R. et al. Germline mutations in the PTEN/MMAC1 gene in patients with Cowden disease. Hum. Mol. Genet. 6, 1383-1387 (1997).

25. Krauthammer, M. et al. Exome sequencing identifies recurrent somatic RAC1 mutations in melanoma. Nat. Genet. 44, 1006-1014 (2012).

26. Watson, I. R. et al. The RAC1 P29S hotspot mutation in melanoma confers resistance to pharmacological inhibition of RAF. Cancer Res. 74, 4845-4852 (2014).

27. Kawazu, M. et al. Transforming mutations of RAC guanosine triphosphatases in human cancers. Proc. Natl Acad. Sci. USA 110, 3029-3034 (2013).

28. Koh, J., Enders, G. H., Dynlacht, B. D. \& Harlow, E. Tumour-derived p16 alleles encoding proteins defective in cell-cycle inhibition. Nature 375, 506-510 (1995).

29. Ranade, K. et al. Mutations associated with familial melanoma impair p16 $6^{\mathrm{INK} 4}$ function. Nat. Genet. 10, 114-116 (1995).

30. Martin, D. W., Munoz, R. M., Subler, M. A. \& Deb, S. p53 binds to the TATA-binding protein-TATA complex. J. Biol. Chem. 268, 13062-13067 (1993).

31. Yan, H. et al. IDH1 and IDH2 mutations in gliomas. New Engl. J. Med. 360, 765-773 (2009).

32. Dang, L. et al. Cancer-associated IDH1 mutations produce 2-hydroxyglutarate Nature 462, 739-744 (2009).

33. Greenman, C. et al. Patterns of somatic mutation in human cancer genomes. Nature 446, 153-158 (2007).

34. Morris, E. J. et al. Discovery of a novel ERK inhibitor with activity in models of acquired resistance to BRAF and MEK inhibitors. Cancer Discov. 3, 742-750 (2013).

35. Lassen, A. et al. Effects of AKT inhibitor therapy in response and resistance to BRAF inhibition in melanoma. Mol. Cancer 13, 83 (2014).

36. Gilmartin, A. G. et al. GSK1120212 (JTP-74057) is an inhibitor of MEK activity and activation with favorable pharmacokinetic properties for sustained in vivo pathway inhibition. Clin. Cancer Res. 17, 989-1000 (2011).

37. Duncan, J. S. et al. Dynamic reprogramming of the kinome in response to targeted MEK inhibition in triple-negative breast cancer. Cell 149, 307-321 (2012).

38. Ussar, S. \& Voss, T. MEK1 and MEK2, different regulators of the G1/S transition. J. Biol. Chem. 279, 43861-43869 (2004).

39. Khokhlatchev, A. et al. Reconstitution of mitogen-activated protein kinase phosphorylation cascades in bacteria. Efficient synthesis of active protein kinases. J. Biol. Chem. 272, 11057-11062 (1997).

40. Sun, C. et al. Reversible and adaptive resistance to BRAF(V600E) inhibition in melanoma. Nature 508, 118-122 (2014).

41. Eblen, S. T. et al. Mitogen-activated protein kinase feedback phosphorylation regulates MEK1 complex formation and activation during cellular adhesion. Mol. Cell. Biol. 24, 2308-2317 (2004).

42. Catalanotti, F. et al. A Mek1-Mek2 heterodimer determines the strength and duration of the Erk signal. Nat. Struct. Mol. Biol. 16, 294-303 (2009).

43. Nathanson, K. L. et al. Tumor genetic analyses of patients with metastatic melanoma treated with the BRAF inhibitor dabrafenib (GSK2118436). Clin Cancer Res. 19, 4868-4878 (2013).

44. Paraiso, K. H. et al. PTEN loss confers BRAF inhibitor resistance to melanoma cells through the suppression of BIM expression. Cancer Res. 71, 2750-2760 (2011).

45. Xing, F. et al. Concurrent loss of the PTEN and RB1 tumor suppressors attenuates RAF dependence in melanomas harboring (V600E)BRAF. Oncogene 31, 446-457 (2012).

46. Rubinstein, J. C. et al. Incidence of the V600K mutation among melanoma patients with BRAF mutations, and potential therapeutic response to the specific BRAF inhibitor PLX4032. J. Transl. Med. 8, 67 (2010).

47. Menzies, A. M. et al. Distinguishing clinicopathologic features of patients with V600E and V600K BRAF-mutant metastatic melanoma. Clin. Cancer Res. 18, 3242-3249 (2012).

48. Falchook, G. S. et al. Dabrafenib in patients with melanoma, untreated brain metastases, and other solid tumours: a phase 1 dose-escalation trial. Lancet 379, 1893-1901 (2012).

49. Ascierto, P. A. et al. Phase II trial (BREAK-2) of the BRAF inhibitor dabrafenib (GSK2118436) in patients with metastatic melanoma. J. Clin. Oncol. 31, 3205-3211 (2013).

50. Long, G. V. et al. Dabrafenib in patients with Val600Glu or Val600Lys BRAFmutant melanoma metastatic to the brain (BREAK-MB): a multicentre, openlabel, phase 2 trial. Lancet Oncol. 13, 1087-1095 (2012).

51. McArthur, G. A. et al. Safety and efficacy of vemurafenib in BRAF(V600E) and $\mathrm{BRAF}(\mathrm{V} 600 \mathrm{~K})$ mutation-positive melanoma (BRIM-3): extended follow-up of a phase 3, randomised, open-label study. Lancet Oncol. 15, 323-332 (2014)

52. Eisenhauer, E. A. et al. New response evaluation criteria in solid tumours: revised RECIST guideline (version 1.1). Eur. J. Cancer 45, 228-247 (2009). 
53. Mann, G. J. et al. BRAF mutation, NRAS mutation, and the absence of an immune-related expressed gene profile predict poor outcome in patients with stage III melanoma. J. Invest. Dermatol. 133, 509-517 (2013).

54. Corcoran, R. B. et al. BRAF gene amplification can promote acquired resistance to MEK inhibitors in cancer cells harboring the BRAF V600E mutation. Sci. Signal. 3, ra84 (2010).

55. Li, H. \& Durbin, R. Fast and accurate short read alignment with BurrowsWheeler transform. Bioinformatics 25, 1754-1760 (2009).

56. DePristo, M. A. et al. A framework for variation discovery and genotyping using next-generation DNA sequencing data. Nat. Genet. 43, 491-498 (2011).

57. Li, H. et al. The Sequence Alignment/Map format and SAMtools. Bioinformatics 25, 2078-2079 (2009).

58. Carlino, M. S. et al. Anti-proliferative effects of continued mitogen activated protein kinase pathway inhibition following acquired resistance to BRAF and/ or MEK inhibition in melanoma. Mol. Cancer Ther. 12, 1332-1342 (2013).

59. Dull, T. et al. A third generation lentivirus vector with a conditional packaging system. J. Virol. 72, 8463-8471 (1998)

60. Haferkamp, S. et al. Oncogene-induced senescence does not require the p16(INK4a) or p14ARF melanoma tumor suppressors. J. Invest. Dermatol. 129, 1983-1991 (2009).

\section{Acknowledgements}

We thank clinicians and staff at Melanoma Institute Australia, Royal Prince Alfred Hospital Department of Pathology and Crown Princess Mary Cancer Centre, Westmead Hospital. We also thank Raghwa Sharma, Megan Lyle, Matthew Chan, Graham J. Mann, Tracy Liaw, Katherine Carson, Valarie Jakrot, Vu Kwan, Peter Smart, Andrew Spillane, Kerwin Shannon, Jonathan Stretch, Libby Emmett, Maria Gonzales, Toni Veale, Kelsey Dobellbrown, Therese Olsson, Anna Hoadley, James Wilmott, Hojabr Kakavand, Chitra De Silva, Tony Neal, Natalie Byrne, Rebecca Hinshelwood, Joanna Jackson, Amie Cho, Vicky Wegener, Karen Byth. R.A.S. and H.R. are supported by the Cancer Institute NSW and NHMRC Fellowship programs. G.V.L. is supported by the Cancer Institute NSW Fellowship program. M.S.C. is supported by Rotary Health Australia scholarship. N.K.H is supported by an NHMRC fellowship. This work is supported by Program Grant 633004 and project grants of the National Health and Medical Research Council of Australia (NHMRC) and an infrastructure grant to Westmead Millennium Institute by the Health Department of NSW through Sydney West Area Health Service. Westmead Institute for Cancer Research is the recipient of capital grant funding from the Australian Cancer Research Foundation.

\section{Author contributions}

G.V.L. and H.R. designed the study. C.F., A.M.M., G.M.P., M.S.C., H.S., V.T., R.A.S. and H.R. performed the experiments. G.V.L., A.M.M., M.S.C., N.K.H., P.J., R.A.S., R.F.K. and H.R. analysed the data. G.V.L., A.M.M., M.S.C., J.F.T., R.P.S., J.H., R.A.S. and R.F.K recruited the patients and provided the reagents/tissues. All authors contributed to the final version of the paper.

\section{Additional information}

Accession codes: Exome sequence data derived from pre-treatment and Prog melanoma tumours have been deposited in the European Nucleotide Archive core database under the accession code PRJEB7503. The microarray data have been deposited in the Gene Expression Omnibus public database at the National Center for Biotechnology Information, under the accession code GSE61992.

Supplementary Information accompanies this paper at http://www.nature.com/ naturecommunications

Competing financial interests: A.M.M.-travel Support, GlaxoSmithKline; Honoraria GlaxoSmithKline; M.S.C.-Honoraria GlaxoSmithKline and Novartis; J.F.T.-travel support, GlaxoSmithKline, Provectus; Consultant Advisor-Roche, GlaxoSmithKline, Provectus; Honoraria-GlaxoSmithKline, Provectus; R.F.K.-Consultant Advisor Roche, GlaxoSmithKline and Novartis; R.A.S.-Consultant Advisor Roche, GlaxoSmithKline and Novartis; G.V.L.-Consultant Advisor Roche, GlaxoSmithKline and Novartis; travel support, Roche; Honoraria Roche, GlaxoSmithKline. The remaining authors declare no competing financial interests.

Reprints and permission information is available online at http://npg.nature.com/ reprintsandpermissions/

How to cite this article: Long, G. V. et al. Increased MAPK reactivation in early resistance to dabrafenib/trametinib combination therapy of BRAF-mutant metastatic melanoma. Nat. Commun. 5:5694 doi: 10.1038/ncomms6694 (2014). 